\title{
Inflammatory Responses in Liver Transplantation
}

\author{
Kantoku Nagakawa, Akihiko Soyama, Susumu Eguchi*
}

Department of Surgery, Nagasaki University Graduate School of Biomedical Sciences, Nagasaki, Japan

\author{
*Corresponding author: \\ Susumu Eguchi, MD, PhD \\ Department of Surgery \\ Nagasaki University Graduate School \\ of Biomedical Sciences, 1-7-1 \\ Sakamoto, Nagasaki 852-8501 \\ Japan \\ Tel: +81-95-819-7316 \\ Fax: +81-95-819-7319 \\ E-mail: sueguchi@nagasaki-u.ac.jp
}

Successful liver transplantation requires proper mitigation of stress responses. Systemic effects caused by liver cirrhosis, invasive surgery, ischemia-reperfusion injury, rejection, and infection, all of which are relevant to liver transplantation, are mediated by inflammatory factors. Recently, the detailed mechanisms of inflammation have been elucidated. Damageassociated molecular patterns (DAMPs) released from cells damaged by ischemia or injury, or pathogen-associated molecular patterns (PAMPs) released by pathogens, are recognized by internal cell receptors or cell surface receptors, leading to the synthesis of inflammatory proteins including proinflammatory/anti-inflammatory cytokines, which are then secreted by the cells. These cytokines stimulate the production of additional cytokines, causing a vicious circle of inflammatory responses, such as innate immune responses by macrophages. In addition, lymphocytes activated by these cytokines stimulate adaptive immunity. During liver transplantation, the general preoperative condition of the patient and surgical invasiveness are associated with organ failure and ischemia-reperfusion injury, which can lead to transplant rejection. It has been suggested that progressive immunodeficiency and organ failure as a result of pre-transplant cirrhosis are associated with postoperative multiple organ failure and death, and that elevated intraoperative DAMPs correlate with organ failure. Furthermore, DAMP receptors and inflammatory cytokines are increased by ischemiareperfusion injury, indicating its role in promoting innate and adaptive immunity. In addition, inflammatory signals induced by postoperative infections are similar to those induced by ischemia-reperfusion injury and rejection, indicating that pathogen-derived inflammation can also lead to rejection. In summary, most complications in liver transplantation can be explained in a unified manner by abnormal inflammatory responses; therefore, controlling inflammation might be of benefit for the struggle of safe and effective liver transplantation.

Key words: inflammation, invasion, liver transplantation

\section{INTRODUCTION}

Successful liver transplantation is a continuous struggle against stress responses. After surgical invasiveness such as a large abdominal incision, removal of the liver, or implanting a graft from another individual, a variety of lethal invasions, including ischemia-reperfusion injury from the graft, organ failure, rejection, and infection from immunosuppressive drugs can occur. These invasions are thought to be different phenomena; therefore, it is extremely difficult to quantify them equally. However, because inflammation might be a
Received: 30.12 .2020

Accepted: 02.03.2021 
unified result of these invasions, it might be used to assess these reactions.

Inflammation is a local tissue reaction induced by harmful stimuli such as pathogens or tissue damage. Cells that receive an initial stimulus are injured and their constituent substances, termed damageassociated molecular patterns (DAMPs), diffuse into the surrounding areas. In contrast, pathogen-associated molecular patterns (PAMPs) are components of pathogens. These DAMPs/PAMPs are initially taken up by phagocytes where they act as intracellular signals to stimulate Toll-like receptors (TLRs) or C-type lectin receptors (CLR), which are patternrecognition receptors (PRRs) on the cell surface, and NOD-like receptors (NLRs), AIM-2 like receptors (ALRs), RIG-I like receptors (RLRs) and receptor for advanced glycation end (RAGE) product, which are intracellular PRRs (1). These receptors are expressed by phagocytes including macrophages, neutrophils, and dendritic cells, as well as epithelial cells, vascular endothelial cells, lung cells, atrium, renal tubules, and gastrointestinal epithelium. Phagocytes and epithelial cells stimulated through PRRs produce cytokines and chemokines via transcription factors such as NF-KB and AP-1 and mRNAs for inflammatory molecules, (2) and by generating a protein complex called the inflammasome that activates caspase-1, which subsequently activates inflammatory cytokines (3). These reactions occur minutes after PRR stimulation. Inflammatory cytokines such as tumor necrosis factor- $\alpha$ (TNF- $\alpha$ ), interleukin (IL)-1 $\beta$, IL-2, IL-6, IL-12, IL-17, IL-18, interferon (IFN)- $\nu$, macrophage migration inhibitory factor (MIF), and chemokines such as $\mathrm{C}-\mathrm{X}-\mathrm{C}$ motif ligand ( $\mathrm{CXCL})-8$ enhance inflammation (fig. 1) (4).

After serum inflammatory cytokines are increased by DAMPs, endothelial cells, including vascular endothelial cells, are activated and express inflammatory receptors, which are involved in multiple organ dysfunction. Thus, DAMPs are mediators of inflammation and initiators of immune responses, because stimulated phagocytes have a main role in innate immunity, leading to the further activation of adaptive immunity (5). In addition, the relationship between inflammation and immunity is complicated because some cytokines are inflammatory and others are anti-inflammatory. On the other hand, all

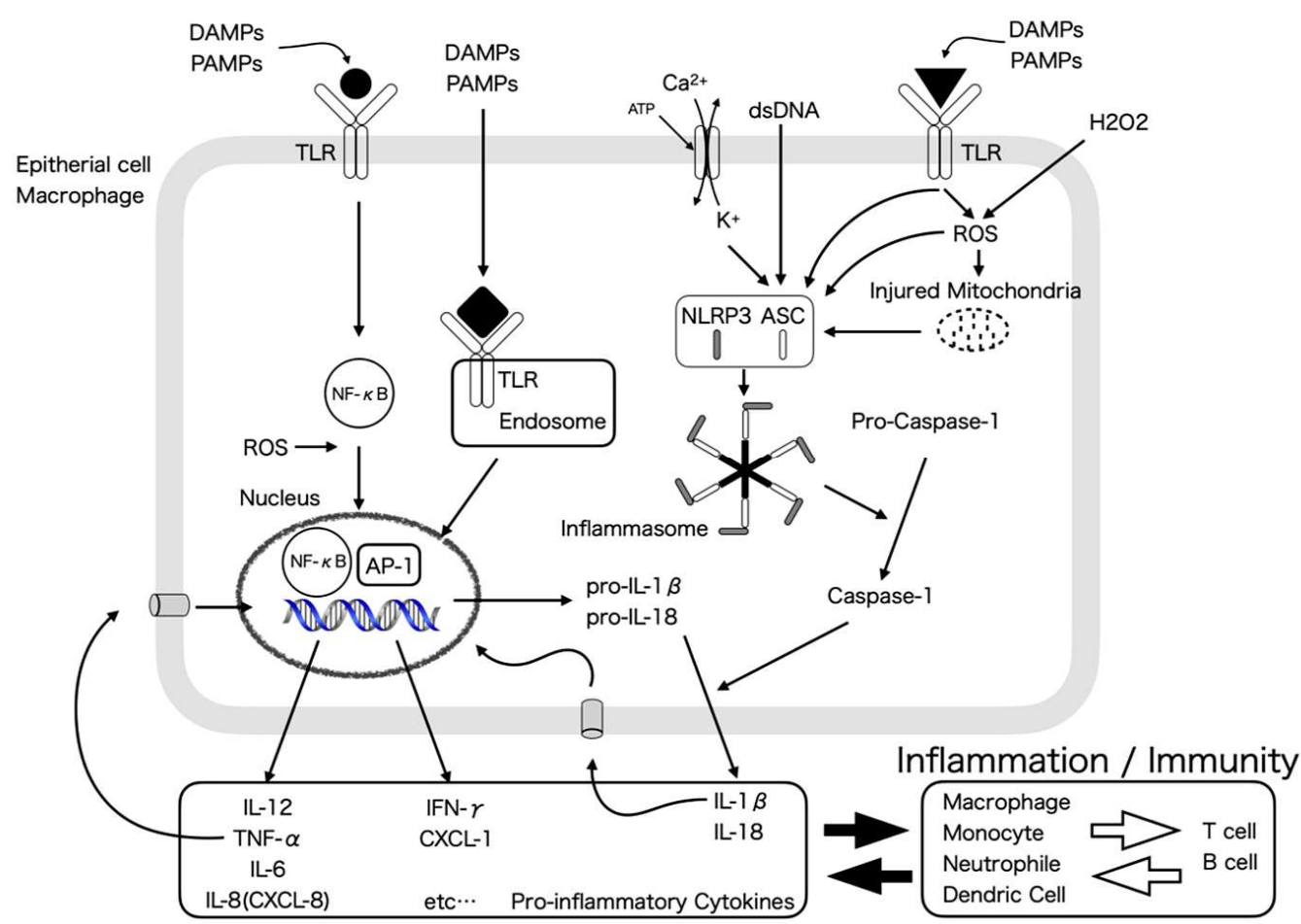

Figure 1 - Inflammatory signal pathway in a cell

TLR signals and ROS activate NF- $\kappa$ B to induce the inflammasome, leading to the transcription of inflammatory cytokines in epithelial cells and macrophages. Proinflammatory cytokines promote inflammation and "turn on" the immune system. DAMPs; damage-associated molecular patterns, PAMPs; pathogen-associated molecular patterns, TLR; Toll-like receptor,

ROS; reactive oxygen species, NF- $\kappa B$; Nuclear factor kappa-light-chain-enhancer of activated B cells, NLRP3; NOD-like receptor family pyrin domain containing 3, ASC; apoptosis-associated speck-like protein containing a C-terminal caspase recruitment domain 
invasions also stimulate the central nervous system, which activates the hypothalamic-pituitary-adrenal pathway, and the sympathetic nervous system, leading to an increase in the secretion of cortisol, catecholamines, and vasopressin. These neuroendocrine systems and inflammatory mediators enhance the metabolic, circulatory, and immune systems to maintain biogenic homeostasis and increase the biogenic defensive capacity (6). Systemic reactions to these responses include fever, tachycardia, tachypnea, and an increase in the number of white blood cells, termed systemic inflammatory response syndrome (SIRS). Therefore, these reactions might be measured by examining the inflammatory responses. In accordance with this concept, this paper describes inflammation during liver transplantation.

\section{Perioperative factors}

Livers with an indication for liver transplantation, have advanced chronic damage, leading to cirrhosis and liver failure. Damage to hepatocytes and cholangiocytes leads to necrosis and apoptosis, and these injured cells release DAMPs, resulting in hypercytokinemia. In response to cytokines, numbers of $\mathrm{CD} 3+\mathrm{T}$ cells, including CD4+ and CD8+ T cells, are increased in the liver and directly damage hepatocytes.(7) In addition, neutrophil chemotactic cytokines, such as cytotoxic CXCL-1 and IL-8 are increased, resulting in an upregulated state that attracts neutrophils, which release proinflammatory cytokines such as TNF- $\alpha$ and IL-17. During this process, hepatic steatosis induced by chemokines such as MCP-1 and CCL2 (also termed "steatokines") and the progression of hepatic fibrosis by the activation of hepatic stellate cells occurs (8). However, during cirrhosis, numbers of endothelial cells are decreased, endothelial cells are dysfunctional, and neutrophil phagocytosis and apoptosis and natural killer cell activity are decreased regardless of hypercytokinemia. This condition is termed cirrhosisassociated immune dysfunction (CAID) (9). In this situation, inflammation occurs in the liver via PAMPs before transplantation. A typical example of a PAMP is lipopolysaccharide (LPS) released by Gram-negative bacteria. Pathogen-derived PAMPs, similar to DAMPs, activate TLRs and induce inflammation, leading to progressive liver injury.

Most patients indicated for liver transplantation have a significant decline in their general condition due to coagulation abnormalities, hepatorenal syndrome, hypotrophy, sarcopenia, or infection related to decreased immunity, caused by decreased hepatic synthetic capacity, or SIRS. Therefore, the preoperative general condition has a significant impact on the intraoperative and postoperative course of the liver transplantation.

We analyzed 60 cases of liver transplantation and found a significant correlation between the preoperative model for end-stage liver disease (MELD) score, and the serum white blood cell (WBC) count $(r=0.46, p<0.01)$, between the pre-operative MELD score and the serum C-reactive protein (CRP) concentration $(r=0.39, p<0.01)$, and between the preoperative MELD score and the serum procalcitonin (PCT) concentration $(r=0.64, p<0.01)$. This indicated that the progression of liver cirrhosis was associated with complications due to infection. In addition, we analyzed the threshold cycle of mitochondrial DNA as a DAMP, which was significantly correlated with the above-mentioned inflammatory findings: WBC $(r=0.53$, $p<0.01), C R P(r=0.44, p<0.01)$, and PCT $(r=0.34, p<0.01)$. WBC and CRP were correlated with PCT regardless of splenomegaly or a decrease of hepatic synthetic function indicating the systemic inflammatory response and susceptibility to infection were a result of the progression of cirrhosis. The correlation between DAMPs and WBC was the greatest of those investigated. The increase in serum DAMPs suggested they acted as inflammatory mediators, and that this might be a useful inflammatory marker. We also analyzed the serum concentrations of DAMPs before laparotomy and at the time of abdominal closure. The serum concentration of DAMPs was increased during surgery and had a weak but significant correlation with the anhepatic time $(r=0.36, p<0.01)$. This result predicted that the magnitude of invasiveness was related to anhepatic time during liver transplantation (10). This finding might be related to the clamping of the inferior vena cava (11), but this was not mentioned in our analysis. Regarding the postoperative disease course, the intraoperative increase of DAMPs was significantly related to postoperative circulatory failure and multiple-organ dysfunction. This suggests that DAMPs are inflammatory mediators that might be treatment targets after liver transplantation.

\section{Ischemia/reperfusion injury}

During organ transplantation, ischemia/reperfusion injury (IRI) is an unpreventable occurrence. IRI is a twostep phenomenon. In the first step, cells are injured by hypoxia due to a decrease in blood flow to the organs, leading to cellular adenosine triphosphate deficiency, acceleration of anaerobic glycolysis, increased lactate 
generation, and alterations in $\mathrm{H}+, \mathrm{Na}+$, and $\mathrm{Ca} 2+$ homeostasis. The second step is exacerbated after the recovery of the oxygen supply. (12) IRI characteristically induces innate immune responses that have an impact on postoperative outcomes. Inflammation due to IRI is promoted by toxic reactive oxygen species (ROS) produced when oxygen is reperfused to ischemic tissues, and is characterized by a cytokine storm, chemokines, growth factors, and DAMPs released by other injured cells. DAMPs released in stressed grafts act as endogenous ligands for PRRs on cells of the innate immune system, such as donor- or recipientderived dendritic cells (DCs) and donor-derived vascular cells. This activates intracellular signaling pathways, leading to the further production of cytokines, chemokines, and growth factors that induce adaptive immune responses that cause graft failure and rejection (13)

Sosa et al. reported changes in the expressions of cytokines and PRRs that recognized DAMPs in the blood during IRI during the perioperative period of liver transplantation, and discussed the main immune response to IRI-related inflammation $(14,15)$. They pathologically diagnosed IRI by intraoperative biopsy and measured circulating blood cytokines before surgery, 1 day, 1 week, and 1 month after surgery, before and after graft passage, and before and after graft reperfusion in IRI and non-IRI cases. In addition, they measured the expression of PRRs during the same timepoints. They found that cases with IRI had higher levels of proinflammatory and anti-inflammatory cytokines preoperatively, with a significant increase in CXCL-8, a neutrophil chemotactic chemokine, which was not related to the background of liver damage or underlying disease, indicating that regardless of the cause, the IRI group had a state of enhanced innate immunity preoperatively. At the time of intraoperative graft perfusion, the concentrations of proinflammatory and anti-inflammatory cytokines in the blood before and after the grafts passed a peak after reperfusion, but the blood concentrations of CXCL-8, which stimulates Th1 cells and lymphocytes, IL-12p70, IL-2, and the anti-inflammatory cytokine, IL-1Ra, were significantly higher in the blood compared with the non-IRI group. The expressions of PRRs tended to increase after graft reperfusion, and TLR4, TLR9, and NOD2, receptors linked to the production of inflammatory cytokines, were increased significantly in the IRI group. The expressions of TLR4 and NOD2 were significantly lower in the IRI group before reperfusion, indicating that these are risk factors for IRI. Similar to the postoperative period, on the first day after transplantation, the levels of antiinflammatory cytokines, IL-1Ra, IL-4, and IL-13, which have Th2 cell-activating effects leading to chronic inflammation, and IL-17A, which has Th17 cell-activating effects, were significantly higher in IRI patients than in non-IRI patients, indicating the start of the adaptive immune response. In addition, high levels of the proinflammatory cytokine IL-1 $\alpha$, but not the anti-inflammatory cytokine IL-1Ra, were also observed, suggesting reactivation of the acute immune response. These results indicate a sustained chronic inflammatory response at this point, in which the innate and adaptive immune responses continued to reactivate each other. One month after transplantation, there was a significant increase in IL-1Ra in the IRI group and IL-1 $\alpha$ levels were no longer significantly different. There was a high level of IL-13, which causes chronic inflammation, and high levels of IL-2, IL-3, and IL-17A, indicating an adaptive immune response and a switch to adaptive immunity (fig. 2). Finally, APCs, antigen-presenting cells and leukocytes activated by these DAMPs and cytokines triggered liver rejection.

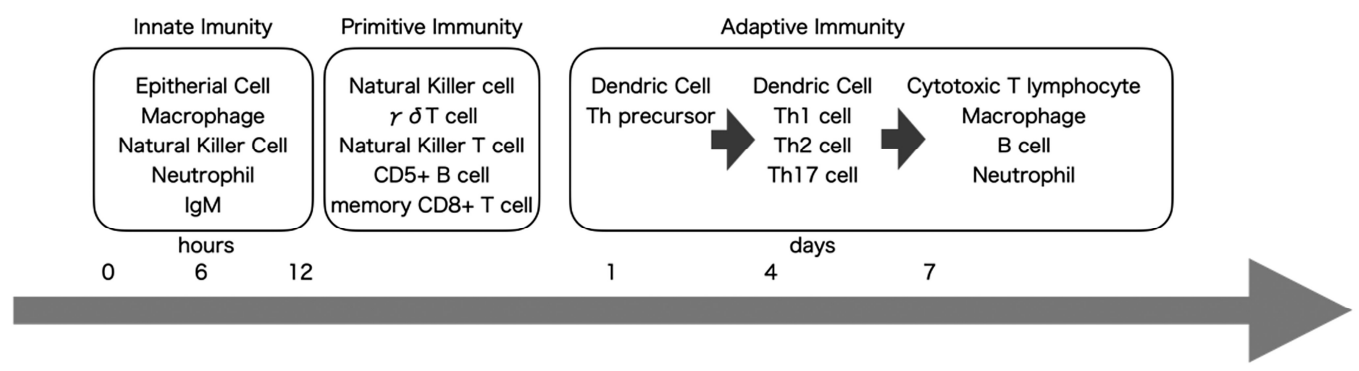

Figure 2 - Sequential change of immunity after transplantation.

After transplantation, ischemic/reperfusion injury affects the recipient. IRI induces immune responses by stimulating innate immune cells. This reaction occurs approximately 12 hours after transplantation. Then, the adaptive immune response is activated. Adaptive immune responses are observed at post-transplantation day 1. IgM; immunoglobulin M, Th; helper T cell. 


\section{Immunological rejection}

As mentioned above, activation of innate immunity due to IRI leads to sterile inflammation and the initiation of acquired immunity. Activated lymphocytes recognize the donor MHC, major histocompatibility complex, of the graft as an antigen, which causes rejection. In parallel to the IRI-induced inflammatory response, donor-derived and recipient-derived DCs take up MHC antigens and are activated after the recognition of various DAMPs by the corresponding PRRs, triggering a robust adaptive anti-donor alloimmune response through a process of direct and indirect antigen recognition, ultimately leading to rejection (13). Indeed, a positive correlation between the frequency of infiltrating macrophages and rejection was reported in renal transplantation (16), and the increased severity of IRI as a result of prolonged ischemic time was shown to increase the risk of acute and chronic graft rejection (17).

Similarly, inflammation has an important role in liver transplantation. Rejection after liver transplantation is usually characterized by inflammatory cell infiltration and damage to bile duct cells and hepatocytes including cellular degeneration and tissue necrosis (18). This suggests there might be a relationship between DAMPs/cytokines and rejection. HMGB-1 is a DAMP that stimulates the production of inflammatory cytokines such as TNF- $\alpha$ and IL-1 $1 \beta$. Increased HMGB-1 levels were observed in the blood of IRI patients after liver transplantation (19), and the association of HMGB-1 with rejection in organ transplantation has been reported.

In a mouse model of MHC mismatch heart transplantation, the pharmacological blockade of HMGB-1 activity reduced graft inflammation and prolonged graft survival by 1 week (20). In a rat versus mouse cardiac xenograft model, HMGB1 expression was upregulated in xenografts and the inhibition of HMGB1 function via anti-HMGB1 monoclonal antibodies prolonged xenograft survival by approximately 1 week and reduced graft inflammation (21). In addition, in chronic allograft rejection in a murine $\mathrm{MHC}$ class II mismatched heart transplant model, HMGB-1 was elevated at 2 months after transplantation, and the administration of anti-HMGB-1 monoclonal antibody every 4 days during the first month after transplantation was associated with a $50 \%$ reduction in the degree of graft vasculopathy, accompanied by a reduction in IL-17A, IFN- $\gamma$, and inflammatory macrophages in the graft (22). These reports suggest that HMGB-1 and its receptor are a major cause of acute and chronic graft rejection.
IL-1 $\beta$ is responsible for acute rejection in organ transplantation $(23,24)$, and HMGB-1 stimulates its production. In liver transplantation, increased levels of IL-1 $\beta$ and the accompanying decrease in receptor antagonists were the main causes of rejection, and elevated receptor antagonists effectively suppressed rejection (25). The production of IL-1 $\beta$ is stimulated by caspase- 1 , which is activated by the inflammasome, containing apoptosis-associated speck-like protein containing a CARD (ASC). In a rat liver transplantation model, the caspase-1 inhibitor group had less rejection from day 1 post-transplantation compared with the no-caspase-1 inhibitor group, and a clear difference was observed at day 7 post-transplantation. The expression of ASC in APCs during this period, the production of IL-1 $\beta$, and hepatocyte necrosis were similar between groups. These results suggest that ASC and upregulation of the inflammatory enzyme activation pathway are closely related to inflammation associated with the acute rejection of transplanted liver (26).

Thus, the relationship between inflammation and rejection is becoming clearer. Currently, most of the immunosuppressive drugs used for liver transplantation are directed at lymphocytes involved in adaptive immunity. In the future, the control of innate immunity might influence the development of new immunosuppressive drugs.

\section{Infection}

In terms of infection, preoperative cirrhosisassociated immune dysfunction (CAID) and postoperative immunosuppression due to surgical invasion and drugs make the recipient susceptible to infection. These effects lead to a state of reduced innate and adaptive immunity, where PAMPs can accelerate inflammation. PAMPs stimulate TLRs, which in turn stimulate the inflammatory cascade. PAMPs that are ligands for TLRs are listed in table 1 (27). In our study, a preoperative PCT $>0.05$ was associated with the incidence of postoperative sepsis (odds ratio $=3.767$, 95\% confidence interval: 1.306-10.866), suggesting it might be a risk factor and predictor of postoperative sepsis (28). The MELD score was also higher in the high preoperative PCT group compared with the low preoperative PCT group. Although a causal relationship is not clear, it may reflect the pathogenesis of CAID. Stimulation of TLRs by PAMPs induced inflammasome activation, IL-1 $\beta$ production, and IRI, leading to acute rejection (29). In terms of cytomegalovirus (CMV) infection, trials using immunoglobulin preparations for CMV prophylaxis in liver transplantation have shown a 
Table 1 - Toll-like receptors and their ligands

\begin{tabular}{|c|c|c|c|c|}
\hline Receptor & Ligand & Ligand location & Location & Cell type \\
\hline TLR1 & Triacyl lipopeptides & Bacteria & Cell surface & PMN, monocyte, macrophage, B cell, DC \\
\hline TLR2 & Glycolipids, LTA, HSP70, zymosan & $\begin{array}{l}\text { Gram-positive bacteria, } \\
\text { cell host }\end{array}$ & Cell surface & Monocyte, myeloid DC, mast cell \\
\hline TLR3 & Double-stranded DNA & Virus & Endosome & $\mathrm{DC}, \mathrm{B}$ cell \\
\hline TLR4 & $\begin{array}{l}\text { Lipopolysaccharide } \\
\text { Heat shock protein } \\
\text { Heparan sulfate fragments } \\
\text { Hyaluronic acid fragments }\end{array}$ & Gram-negative bacteria & Cell surface & $\begin{array}{l}\text { Monocyte, macrophage, myeloid DC, } \\
\text { mast cell, intestinal epithelium }\end{array}$ \\
\hline TLR5 & Flagellin & Bacteria & Cell surface & Monocyte, macrophage, DC, intestinal epithelium \\
\hline TLR6 & Multiple diacyl lipopeptides & Mycoplasma & Cell surface & Monocyte, macrophage, mast cell, B cell \\
\hline TLR7 & $\begin{array}{l}\text { Imidazoquinoline, Loxoribine, } \\
\text { Bropirimine, Single-stranded DNA }\end{array}$ & Small synthetic components & Endosome & Monocyte, macrophage, plasmacytoid DC, B cell \\
\hline TLR8 & $\begin{array}{l}\text { Small synthetic compounds } \\
\text { Single-stranded RNA }\end{array}$ & & Endosome & Monocyte, macrophage, DC, mast cell \\
\hline TLR9 & Unmethylated CpG-DNA & Bacteria & Endosome & Monocyte, macrophage, plasmacytoid DC, B cell \\
\hline TLR10 & Unknown & Unknown & Cell surface & Monocyte, macrophage, B cell \\
\hline TLR11 & Profilin & $\begin{array}{l}\text { Toxoplasma gondii, } \\
\text { uropathogenic Escherichia coli }\end{array}$ & Cell surface & $\begin{array}{l}\text { Monocyte, macrophage, liver cell, kidney cell, } \\
\text { bladder epithelium }\end{array}$ \\
\hline
\end{tabular}

PMN; polymorphonuclear leukocyte, DC; dendritic cell

significant reduction in rejection, suggesting the effect of infection on innate and adaptive immunity (30).

As described above, it is thought that there is a close relationship between infection and rejection because the systems that induce inflammation are the same, and the control of immunosuppressive drugs is important for infection and rejection. Although a few studies have reported a relationship between infection and rejection, the mechanism is poorly understood (31). We await further clarification.

\section{Conclusion}

Although inflammation is a unitary system in liver transplantation, it has multifactorial effects that affect the prognosis of the graft and the recipient. If we can control inflammation, we can enhance liver transplantation.

\section{Financial disclosures}

No external funding resources.

\section{Conflicts of interest}

All authors declare no conflicts of interest.

\section{Ethics of approval}

Ethical approval is not necessary.

\section{REFERENCES}

1. Takeuchi 0 , Akira S. Pattern recognition receptors and inflammation. Cell. 2010;140:805-820.

2. Fujioka S, Niu J, Schmidt C, Sclabas GM, Peng B, Uwagawa T, et al. NF-kappaB and AP-1 connection: mechanism of NF-kappaBdependent regulation of AP-1 activity. Mol Cell Biol. 2004;24(17): 7806-19.

3. Szabo G, Petrasek J. Inflammasome activation and function in liver disease. Nat. Rev. Gastroenterol. Hepatol. 2015;12:387-400.

4. Matsumoto $\mathrm{H}$, Ogura $\mathrm{H}$. Inflammatory cytokine. In Matsuda N (eds.), Inflammatory response and coagulation/fibrinolysis system in severe patient. Nakayama Shoten Co, Ltd. 2017;67-79.

5. Zhang $Q$, Raoof $M$, Chen $Y$, Sumi $Y$, Sursal $T$, Junger $W$, et al. Circulating mitochondrial DAMPs cause inflammatory responses to injury. Nature. 2010;464:104-107.

6. Bonaz B, Sinniger V, Pellissier S. Anti-inflammatory properties of the vagus nerve: potential therapeutic implications of vagus nerve stimulation. J Physiol. 2016;594:5781-5790.

7. Gao B, Ahmad MF, Nagy LE, Tsukamoto H. Inflammatory pathways in alcoholic steatohepatitis. J Hepatol. 2019;70(2):249-259.

8. Huang H, Tohme S, Al-Khafaji AB, Tai S, Loughran P, Chen L, et al. Damage-associated molecular pattern-activated neutrophil extracellular trap exacerbates sterile inflammatory liver injury. Hepatology. 2015;62(2):600-14.

9. Bonnel AR, Bunchorntavakul C, Reddy KR. Immune dysfunction and infections in patients with cirrhosis. Clin Gastroenterol Hepatol. 2011;9(9):727-38.

10. Nagakawa K, A Soyama, M Hidaka, Adachi T, Ono S, Hara T, et al. Elevated plasma levels of mitochondria-derived damage-associated molecular patterns during liver transplantation: predictors for postoperative multi-organ dysfunction syndrome. Tohoku J Exp Med. 2020;250(2):87-93.

11. Shih TH, Chen CC, Chen CL, Huang CJ, Cheng KW, Wang CH, et al. Acute kidney injury in adult living donor liver transplantation performed with different methods of inferior vena cava clamping maneuvers. Transplant Proc. 2020;52(6):1849-1851.

12. Zwacka RM, Zhang $Y$, Halldorson J, Schlossberg $H$, Dudus $L$, Engelhardt JF. CD4(+) T-lymphocytes mediate ischemia/reperfusioninduced inflammatory responses in mouse liver. J Clin Invest. 1997; 100(2):279-89. 
13. Lu L, Zhou H, Ni M, Wang X, Busuttil R, Kupiec-Weglinski J, et al. Innate immune regulations and liver ischemia-reperfusion injury. Transplantation. 2016;100(12):2601-2610.

14. Sosa RA, Zarrinpar A, Rossetti M, Lassman CR, Naini BV, Datta N, et al. Early cytokine signatures of ischemia/reperfusion injury in human orthotopic liver transplantation. JCI Insight. 2016;1(20): e89679.

15. Sosa RA, Rossetti M, Naini BV, Groysberg VM, Kaldas FM, Busuttil RW, et al. Pattern recognition receptor-reactivity screening of liver transplant patients: potential for personalized and precise organ matching to reduce risks of ischemia-reperfusion injury. Ann Surg 2020;271(5):922-931.

16. Brasen JH, Khalifa A, Schmitz J, Dai W, Einecke G, Schwarz A, et al. Macrophage density in early surveillance biopsies predicts future renal transplant function. Kidney International. 2017;92:479-489.

17. Gjertson, D. Impact of delayed graft function and acute rejection on kidney graft survival. Clin. Transpl. 2000;467-480.

18. Sis B, Mengel M, Haas M, Colvin RB, Halloran PF, Racusen LC, et al . Banff '09 meeting report: antibody mediated graft deterioration and implementation of Banff working groups. Am J Transplant. 2010 10(3):464-71.

19. van Golen RF, Reiniers MJ, Marsman G, Alles LK, van Rooyen DM, Petri B, et al. The damage-associated molecular pattern HMGB1 is released early after $T$ clinical hepatic ischemia/ reperfusion. Biochim Biophys Acta Mol Basis Dis. 2019;1865(6): 1192-1200.

20. Huang $Y$, Yin $H$, Han J, Huang B, Xu J, Zheng F, et al. Extracellular hmgb1 functions as an innate immune-mediator implicated in murine cardiac allograft acute rejection. Am J Transplant. 2007; 7(4):799-808.

21. Li JH, Zhao B, Zhu XH, Wang L, Zou HJ, Chen S, et al. Blockade of extracellular HMGB1 suppresses xenoreactive $B$ cell responses and delays acute vascular xenogeneic rejection. Am J Transplant. 2015 15(8):2062-74.

22. Zou H, Yang $Y$, Gao M, Zhang B, Ming B, Sun $Y$, et al. HMGB1 is involved in chronic rejection of cardiac allograft via promoting inflammatory-like mDCs. Am J Transplant. 2014:14(8):1765-77.

23. Wanderer AA. Rationale and timeliness for IL-1 beta-targeted therapy to reduce allogeneic organ injury at procurement and to diminish risk of rejection after transplantation. Clin Transplant. 2010; 24:307-311.

24. Obara H, Nagasaki K, Hsieh CL, Ogura Y, Esquivel CO, Martinez OM, et al. IFN-gamma, produced by NK cells that infiltrate liver allografts early after transplantation, links the innate and adaptive immune responses. Am J Transplant. 2005;5(9):2094-103.

25. Liu Z, Yan L, Li S, You H-b, Gong J-p. Glycine blunts transplantative liver ischemia-reperfusion injury by downregulating interleukin 1 receptor associated kinase-4. Acta Pharmacol Sin. 2006;27(11): 1479-86.

26. Hong BJ, Liu $H$, Wang ZH, Zhu YX, Su LY, Zhang MX, et al. Inflammasome activation involved in early inflammation reaction after liver transplantation. Immunol Lett. 2017;190:265-271.

27. Frazăo JB, Errante PR, Condino-Neto A. Toll-like receptors' pathway disturbances are associated with increased susceptibility to infections in humans. Arch Immunol Ther Exp (Warsz). 2013;61: 427-443.

28. Hara T, Soyama A, Hidaka M, Natsuda K, Adachi T, Ono S, et al. Pretransplant serum procalcitonin level for prediction of early posttransplant sepsis in living donor liver transplantation. Hepatol Res. 2018;48(5):383-390.

29. Weigt SS, Palchevskiy V, Belperio JA. Inflammasomes and IL-1 biology in the pathogenesis of allograft dysfunction. J Clin Invest. 2017; 127:2022-2029.

30. Kornberg A, Witt U, Kornberg J, Müller K, Friess H, Thrum K. Prophylactic anti-cytomegalovirus hyperimmunoglobulin in critically ill liver transplant patients: impact on early immunology and survival. J Clin Med. â Feb 29;9(3):656.

31. Sánchez-Ponce $Y$, Varela-Fascinetto G, Romo-Vázquez JC, LópezMartínez B, Sánchez-Huerta JL, Parra-Ortega I, et al. Simultaneous Detection of Beta and Gamma Human Herpesviruses by Multiplex qPCR Reveals Simple Infection and Coinfection Episodes Increasing Risk for Graft Rejection in Solid Organ Transplantation. Viruses. 2018;10(12):730. 